\title{
Automated High-Temperature PVT Apparatus With Data for Propane
}

\author{
G. C. Straty \\ National Bureau of Standards, Boulder, CO 80303 \\ and \\ A. M. F. Palavra \\ Instituto Superior Tecnico, Lisboa-1, Portugal
}

Accepted: June 20, 1984

\begin{abstract}
An apparatus is described which can be used for PVT and compressibility measurements on supercritical fluids from near room temperature to $600^{\circ} \mathrm{C}$ and pressures to $35 \mathrm{MPa}$. Two separate experimental techniques are employed to obtain PVT data over a broad range of the state surface. Burnett expansions are performed to generate compressibility factor (or equivalently density) data along a well-behaved supercritical isotherm. A series of isochoric measurements is then made to extend the temperature range. Densities assigned to the isochores are determined from their intersection with the previously measured Burnett isotherm or gravimetrically. A computer is used for experimental control and for data logging. Isochoric measurements lasting several days can be performed routinely and without operator attention. The apparatus has been tested on propane to a temperature of $325^{\circ} \mathrm{C}$. The density data, estimated accurate to \pm 0.1 percent, are in excellent agreement with other existing data.
\end{abstract}

Key words: Burnett method; compressibility; density; fluids; propane; PVT behavior.

\section{Introduction}

Perhaps the single most important property of any compressible fluid is its PVT behavior. An accurate and precise description of the PVT surface can, in principle, be used to calculate most equilibrium properties. PVT data are also required to analyze experimental data on other quantities and to perform engineering design calculations on fluid handling systems. Wide range PVT data for the many technically important fluids at

About the Authors, Paper: G. C. Straty is with the Chemical Engineering Science Division of the NBS National Engineering Laboratory, while A. M. F. Palavra serves with the Centro de Quimca Estrutural, Complexo Interdisciplinar, in Portugal's Instituto Superior Tecnico. The work they report on was carried out for the Department of Energy, Office of Basic Energy Sciences. elevated temperatures are, however, often scarce and in some cases are nonexistent.

We describe here an experimental apparatus that can be used for PVT determinations on fluids above room temperature. Two separate experimental techniques are used to obtain data over a broad range of the state surface (see fig. 1). The well known Burnett $[1]^{1}$ method of successive gas expansions is used to establish the density behavior for the fluid along a well-behaved supercritical isotherm. The maximum temperature of such measurements is, however, limited by such things as the compatibility of valve packings and the pressure transducer with the elevated temperatures. The measurement of pressure versus temperature along a nearly isochoric path (pseudo-isochores) in a second experiment is then used to extend the temperature range. Densities assigned to the pseudo-isochores can be determined from their intersection with the previously measured isotherms.

\footnotetext{
${ }^{1}$ Figures in brackets indicate literature references and explanatory notes at the end of this paper.
} 


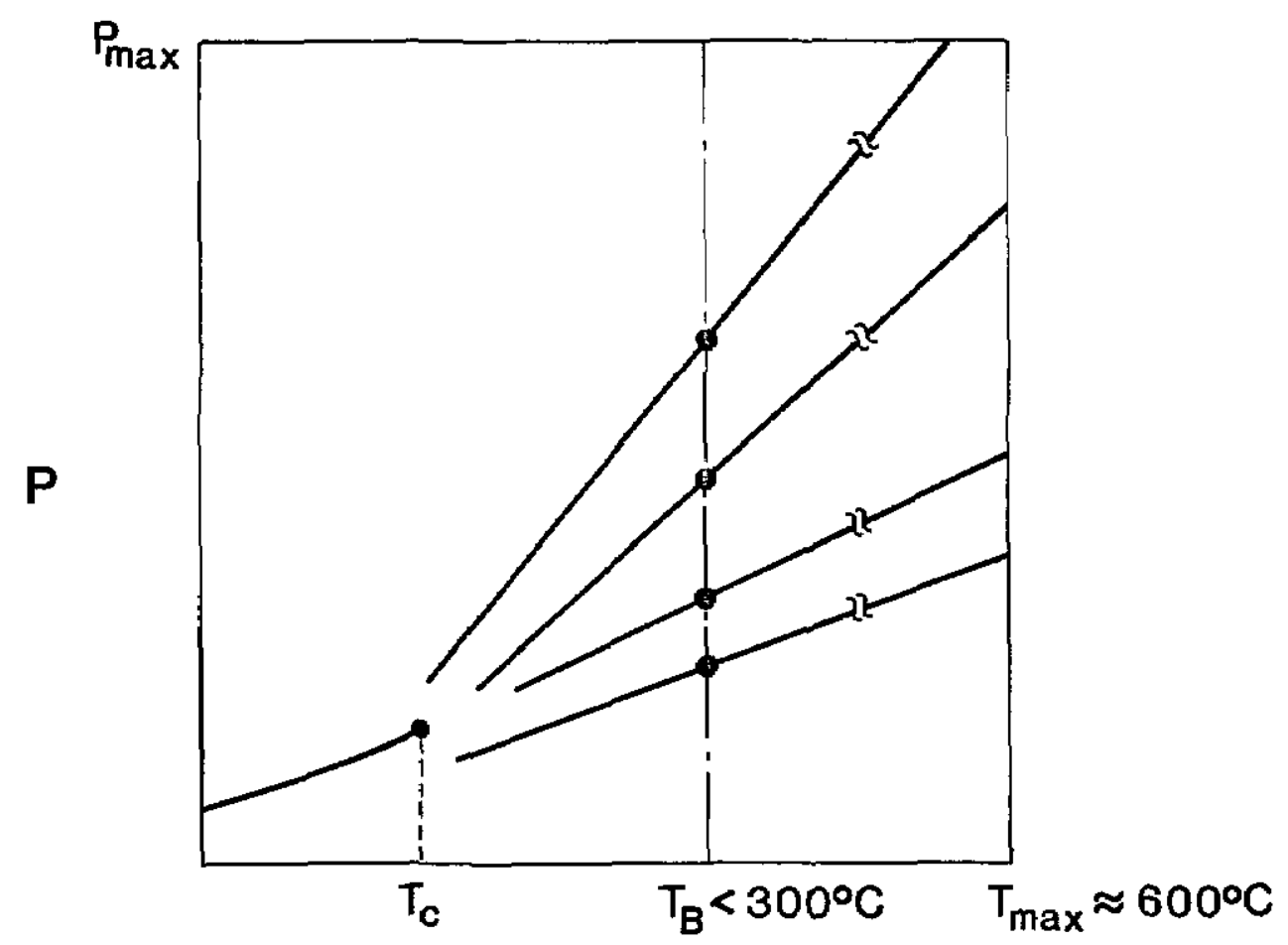

Figure 1-Schematic of the P-T plane for a typical fluid. Densities along the $T_{B}$ isotherm are obtained using a Burnett expansion technique. $P-T$ measurements along pseudo isochores are then made to extend the temperature range from near critical temperature to $T_{\max }$. Isochore densities are obtained from their intersection with the $T_{B}$ isotherm.

\section{T}

Principal objectives in the design of the apparatus were: 1) the apparatus should be capable of measurements covering a broad temperature and pressure range, 2) the determined densities should be independent of the data accuracy for any calibration fluid, and 3) the apparatus should be automated as much as possible to minimize the necessity for operator attention.

The apparatus is capable of operation from near room temperature to about $600^{\circ} \mathrm{C}$ at pressures to over 35 MPa. Densities can be determined by using two completely independent techniques, the Burnett method involving successive fluid expansions, or a gravimetric technique involving only the well known density of water used for volume calibration purposes. A small desktop computer is employed for experimental control, data logging, and partial real time data analysis. Measurement runs along isochores taking days to complete can be performed automatically and without operator attention.

\section{Experimental}

The experimental apparatuses are illustrated schematically in figure 2 . The Burnett system [shown in a) and b) of fig. 2] consists of three spherical cells of different volumes and a diaphram type differential pressure transducer with valving as shown. The cells with nomi- nal volumes of about 200,120 and $40 \mathrm{~cm}^{3}$ allow different volume ratios to be selected. These elements are suspended from a thermally insulated platen which forms the top closure to a thermostated bath used for temperature control. This arrangement is similar to that described previously in detail [2].

Mixed liquid baths are usually preferred and have traditionally been used for thermostating in this type of experiment. Because of the elevated temperatures of this work, however, a commercially available oven with vigorous circulation was modified for the purpose. Air is normally used as the convective heat transfer fluid but, for safety reasons, a continuous gas purge (typically nitrogen) is employed for work on flammable samples.

Even though the heat transfer gas is vigorously mixed, substantial temperature differences can exist over the oven volume. To minimize these differences, the components are encased in a massive aluminum block [not shown in a) of fig. 2 for clarity]. The typical arrangement is shown in b) of figure 2. Space is provided around each component to partially decouple it thermally from the block and to permit free flow of the heat transfer gas around the components. This arrangement reduces temperature differences to a few tenths of a degree. Further reduction in temperature differences is accomplished by using very low power shimming 
Fill-Vac

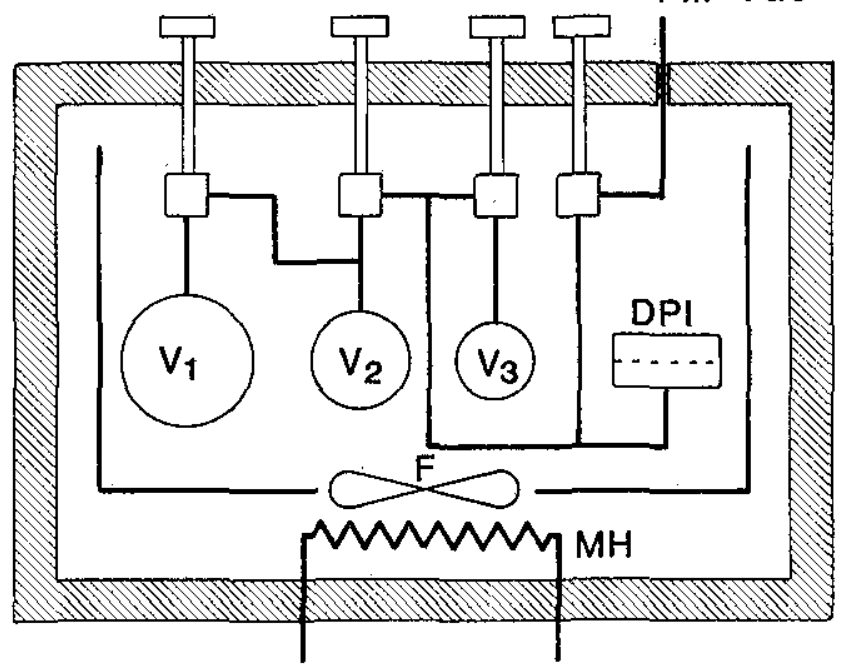

a)

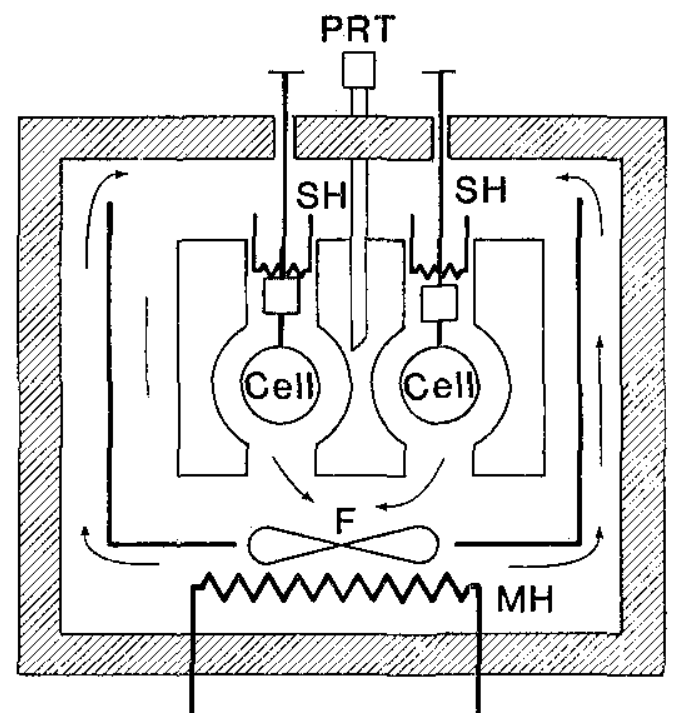

b)

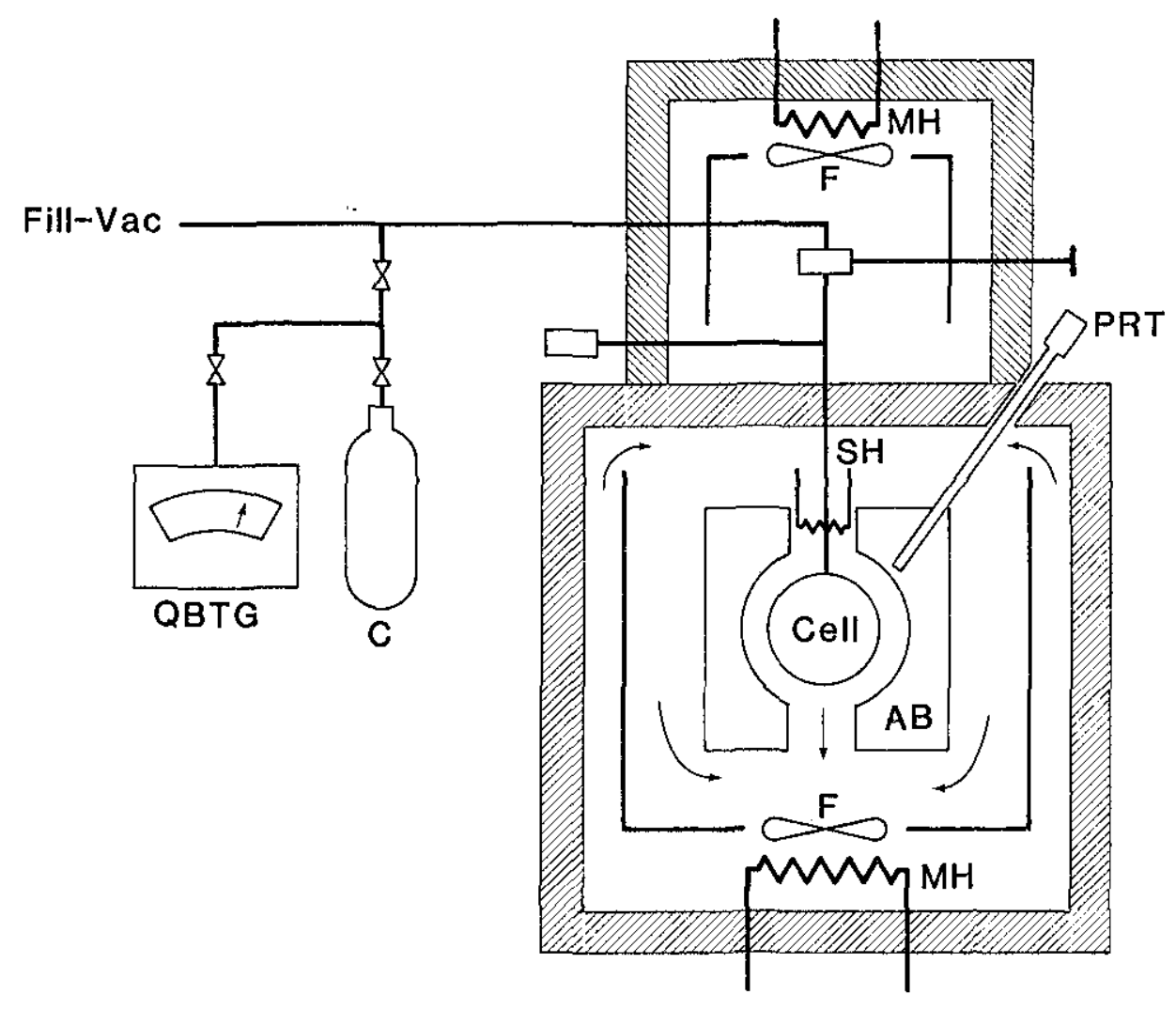

c)

Figure 2-Schematic of the apparatuses. a) Burnett system showing cells, valving and piping. b) Burnett system showing the arrangement for temperature control. c) Isochoric system. In a), $V_{1}, V_{2}, V_{3}=$ three spherical cells and DPI=differential pressure transducer. In a), b), c), $\mathrm{F}=$ forced convection fan and $\mathrm{MH}=$ main heaters. In b) and $\mathrm{c}$, $\mathrm{SH}=\mathrm{shim}$ heaters, $\mathrm{PRT}=$ platinum resistance thermometer and $\mathrm{AB}=$ aluminum block. In $\mathrm{c}$ ), $\mathrm{QBTG}=$ quartz bourdon tube gauge (pressure) and $\mathrm{C}=$ condensate cell. 
heaters located in the air stream above each major component.

By means of diaphram type differential pressure transducers, pressures in the Burnett experiment are measured by referencing to oil pressure derived from an oil operated commercial pressure balance with a stated accuracy of $0.015 \%$.

A spherical configuration was chosen for the cells to allow a more accurate calculation of the change in cell volumes with pressure. Each cell was constructed with an identical ratio of outside to inside diameter to provide maximum error cancellation in the calculated volume ratios.

The system employed for the isochoric measurements is illustrated in c) of figure 2. This system consists of a single spherical cell with nominal volume of $120 \mathrm{~cm}^{3}$, encased in an aluminum block and suspended from a second insulated platen. To change experiments the Burnett system is simply replaced in the oven by the isochoric system.

Since the isochoric measurements can be made up to the maximum temperature of $600^{\circ} \mathrm{C}$, it is necessary to locate the valve and the pressure transducer in a less hostile environment. The valve and piping are enclosed in a second oven, maintained at the temperature of the initial Burnett measurements, where the density has already been determined, to allow a more accurate correction to be made to the assigned densities along the isochores.

Pressures along the isochores are determined from the vibration frequency of a commercial 0-40 MPa quartz transducer, calibrated frequently against the primary dead weight pressure balance. The transducer is estimated to be accurate to better than $0.05 \%$ full scale. The pressure transducer is located outside of the ovens in an enclosure thermostated at $40^{\circ} \mathrm{C}$.

Volumes of the isochoric cell, piping, valve, and pressure transducer were calibrated by low pressure nitrogen gas expansions from a volume previously calibrated by water weighing. This procedure allows isochore densities to be determined by weighing the sample after a run and provides a completely independent check on the densities determined in the gas expansion measurements.

Temperatures in both experiments are measured by using a platinum resistance thermometer (PRT) calibrated on the IPTS- 68 by the National Bureau of Standards. The PRT is used to measure the temperature of the aluminum block. Temperatures of the components are then calculated from the block temperature and from any small temperature differences existing between the block and the components as determined by suitably placed differential thermocouples.
In use, either apparatus is suspended from a supporting structure. A mechanism for manipulating the ovens to enclose the apparatus for an experimental run and to retract them to provide access to the components is provided. For safety reasons, when working with toxic materials, the support structure is enclosed in a manner similar to a ventilation hood and is continuously aspirated by an exhaust fan located on the laboratory roof.

\section{Computer Control and Data Acquisition}

A computer was used for both experiment control and data acquisition. The series of Burnett measurements necessarily involved manual valve and pressure balance manipulations. In this case, the computer functions primarily as a thermal controller to bring the various elements of the apparatus into thermal equilibrium at the same temperature. The PRT and thermocouples are interrogated under computer control at a programmed time interval, usually about $30-40 \mathrm{~s}$, using a scanner of custom design. Scanner relays were purchased commercially and had a claimed thermal EMF of less than $0.02 \mu \mathrm{V}$. Potentials are measured with a DVM with nanovolt resolution. Primary thermometry is performed by taking the ratio of the potential across the PRT and the potential across a series connected standard resistance of $50 \Omega$, carrying a current of less than $0.001 \mathrm{~A}$, using a single DVM for the measurement. Two ratio determinations are made for each temperature measurement with current reversed for each. Averaging the two measurements minimizes the errors due to residual thermal EMF and tends to cancel DVM zero offsets. Temperature differences between the various apparatus components, calculated by the computer from the measured differential thermocouple potentials, are used to activate the shimming heaters to bring all elements to the target temperature. In equilibrium, temperature differences are typically maintained to better than $\pm 0.025^{\circ} \mathrm{C}$ at $250^{\circ} \mathrm{C}$. Since corrections are made for these temperature differences in the data analysis, differences of as much as $0.1{ }^{\circ} \mathrm{C}$ are considered tolerable. Data are logged onto hard copy and cassette tape after equilibrium is achieved, as determined by the operator.

The $P-T$ measurements along pseudo-isochores are performed almost completely under computer control. Only initial filling of the system and subsequent venting or weighing of the sample at the end of a run is required of the operator.

In operation, the computer selects the preprogrammed temperature, sets the oven temperature and then determines the block temperature, cell temperature, and the rate of approach of the cell to the 
target temperature by interrogating the various thermocouples and the PRT at programmed intervals of about $30-40 \mathrm{~s}$. When the cell temperature is within $0.1{ }^{\circ} \mathrm{C}$ of the target temperature, the computer establishes a holding mode where the temperature control is continuously refined. Temperatures normally oscillate only a few hundredths of a degree about the target temperature in this mode. After an equilibration time (usually one hour) the data are logged onto hard copy and cassette tape, but only if programmed limits of temperature deviation and temperature and pressure drift rates are satisfied. The experiment is automatically shut down after a preset maximum pressure or temperature is reached. The sample is then manually vented, or it can be condensed into a small cell maintained at liquid nitrogen temperature for subsequent weighing.

Isochoric runs on propane at temperatures up to $325^{\circ} \mathrm{C}$, taking $50-60 \mathrm{~h}$ to complete, were carried out completely without operator attention. An automatic dial-up smart MODEM is interfaced with the controlling computer and allows experimental progress to be monitored and controlled by telephone from any remote site using a portable terminal or personal computer. Experiments usually are run continuously including evenings and weekends.

\section{Data Analysis}

The usual Burnett experiment involves the expansion of the sample gas from an initial volume $V_{1}$ into a second evacuated volume $V_{2}$. Pressures before and after the expansion are measured. After isolation of $V_{1}$ and evacuation of $V_{2}$, the process is repeated successively to low pressure. For any particular expansion.

$$
\frac{P_{i}}{P_{i+1}}=\frac{Z_{i}}{Z_{i+1}} N_{i}
$$

where $\quad P=$ pressure, $\quad Z=\frac{P}{\rho R T}, \quad \rho=$ density $\quad$ and $N_{\mathrm{i}}=\frac{V_{1}+V_{2}}{V_{1}}$. It is assumed here that the temperatures of $V_{1}$ and $V_{2}$ are constant and identical and it is recognized that the volume ratio $N_{i}$ is slightly pressure dependent.

A number of ways to analyze such data have been suggested [4-6]. One common way is to express the compressibility factor $Z$ as some function of pressure

$$
Z=F(P) .
$$

Least squares analysis can be applied to the experimental data using eq (1) to determine the volume ratio $N_{0}$ at zero pressure and the parameters of $F(P)$. If $F(P)$ is a virial equation the determined parameters can be identi- fied with the virial coefficients provided that the range of the data falls within the region where the virial equation is valid and the proper number of terms have been retained in the virial series. Considerable attention has been devoted to the problem of how to determine the proper number of terms to retain in the virial expansion [4-6]. The object of this work, however, is to obtain densities. We avoid the problem of the virial coefficients and use $F(P)$ only as a fitting function. The method can usually be used with data up to a maximum pressure corresponding to a density somewhat less than the critical density. Densities for higher pressure points can be obtained from the lower pressure data using the cell constant since

$$
\rho_{i}=\left(\rho_{i+1}\right) N_{i}
$$

Even though it is possible to obtain the cell constant $N_{0}$ from a single fit to the experimental data, it is common, and often more accurate, to obtain it from a separate calibration experiment using a highly ideal fluid, usually helium. Extrapolation of the pressure ratio $P_{i} / P_{i+1}$ to zero pressure yields the volume ratio directly since $Z$ is identically one at zero pressure. A major advantage of the Burnett technique is that it is not necessary to do extensive, individual volume calibrations on the system, since only the volume ratio, which can be obtained from the expansion data, is required for data analysis.

For this work, the analysis is complicated by the fact that small temperature differences are allowed to exist between the volume elements composing the system. Equation (1) becomes

$$
\frac{P_{i}}{P_{i+1}}=\sum_{j=1}^{n} \frac{V_{i+1, j}}{V_{i, 1}} \frac{T_{i, 1}}{T_{i+1, j}} \frac{Z_{i, 1}}{Z_{i+1, j}}
$$

where the system is now considered to consist of $n$ volume elements which include the primary volumes $V_{1}$ and $V_{2}$ plus the volumes of connecting piping, valves and pressure transducer all at slightly different temperatures. The particular volume element is identified by the second subscript while the first subscript indicates the particular expansion as before. In general it is no longer sufficient for accurate data analysis to know only the cell constant $N_{0}$; the magnitude of all volume elements must also be known individually as well as certain density derivatives of the state surface.

For this analysis, the concept of a cell constant is retained but now

$$
N_{0}=\sum_{j=1}^{n} \frac{V_{0, j}}{V_{0,1}}
$$


where the subscript 0 again indicates $P_{i}=0$ and all volume elements are at the target temperature $T$. The $V_{i, j}$ can be expressed in terms of the $V_{0, J}$ as

$$
V_{i, j}=V_{0, j}\left(1+\alpha \Delta T_{i, j}\right)\left(1+\beta_{j} P_{i}\right),
$$

where $\alpha=$ volume thermal expansion coefficient, $\beta_{j}=$ pressure dilation coefficient for volume $j$ and the $\Delta T_{i, j}=T_{i, j}-T$ are the deviations from the target temperature $T$. If the $\Delta T \ll T$ and recognizing the fact that

$$
\lim _{P \rightarrow 0} \frac{\left(1+\beta_{j} P_{i+1}\right)}{\left(1+\beta_{1} P_{i}\right)} \frac{Z_{i, 1}}{Z_{i+1, j}}=1
$$

eq (4) can be used to obtain, after some manipulation and small approximation,

$N_{0} \simeq \lim _{P \rightarrow 0}\left(1+\alpha \Delta T_{i, 1}\right) \frac{T}{T_{l, 1}} \frac{P_{i}}{P_{i+1}}+\left(\frac{1}{T}-\alpha\right) \sum_{j=1}^{n} \frac{V_{0, i}}{V_{0,1}} \Delta T_{i+1, j}$.

Equation (8) is used to obtain the cell constant $N_{0}$ using calibration data measured with helium. The first term on the right contains measured quantities. The second term is of order $10^{-4} N_{0}$ which allows the use of calculated values for the individual volume ratios $\left(V_{0, j} / V_{0,1}\right)$ without introducing significant error in the value of $N_{0}$ obtained.

Using eq (6) in eq (4) the expression describing any expansion becomes

$$
\frac{P_{i}}{P_{i+1}}=\frac{Z_{i, 1} T_{i, 1}}{1+\alpha \Delta T_{i, 1}+\beta_{1} P_{i}} \sum_{j=1}^{n} \frac{V_{0, j}}{V_{0,1}}\left(\frac{1+\beta_{j} P_{i+1}+\alpha \Delta T_{i+1, j}}{Z_{i+1, j} T_{i+1, j}}\right),
$$

where the very small terms in $\alpha \beta$ have been ommitted for clarity but which are included in the actual data reduction programs. For $\Delta T \ll T$ it is assumed that $\frac{\partial Z_{i, j}}{\partial T_{i, j}}=\frac{\partial Z_{i}}{\partial T}$ and the compressibility factor $Z_{i}$ at the target temperature $T$ is related to $Z_{i, j}$ by

$$
Z_{i, j}=Z_{i}\left[1+\left.\frac{1}{Z_{i}} \frac{\partial Z_{i}}{\partial T}\right|_{P} \Delta T_{i, j}\right] .
$$

Equation (9) can then be written, again after some manipulation, as

$$
\begin{gathered}
\frac{P_{i}}{P_{i+1}}=\frac{Z_{i}}{Z_{i+1}}\left[1+\left.\frac{1}{Z_{i}} \frac{\partial Z_{i}}{\partial T}\right|_{p} \Delta T_{i, 1}\right] \\
\left(\frac{T_{i, 1}}{T}\right)\left(\frac{A+P_{i+1} B+\alpha C}{1+\beta_{1} P_{i}+\alpha \Delta T_{i, 1}}\right),
\end{gathered}
$$

where

$$
A=N_{0}+\left.\frac{1}{\rho_{i+1}} \frac{\partial \rho_{i+1}}{\partial T}\right|_{p} \sum_{j=1}^{n} \frac{V_{0, i}}{V_{0,1}} \Delta T_{i+1, j}
$$

$$
B=\sum_{j=1}^{n} \frac{V_{0, j}}{V_{0,1}} \beta_{j}+\left.\frac{1}{\rho_{i+1}} \frac{\partial \rho_{i+1}}{\partial T}\right|_{p j=1} ^{n} \frac{V_{0, j}}{V_{0,1}} \beta_{j} \Delta T_{i+\mathrm{t}, j}
$$

and

$$
C=\sum_{j=1}^{n} \frac{V_{0, j}}{V_{0,1}} \Delta T_{i+1, j}+\left.\frac{1}{\rho_{i+1}} \frac{\partial \rho_{i+1}}{\partial T}\right|_{P=1} ^{n} \frac{V_{0, j}}{V_{0,1}} \Delta T_{i+1, j}^{2} .
$$

For $\Delta T=0$ this reduces to the isothermal Burnett situation. The $N_{0}$ in eq (12) is the largest term and is determined from the helium calibration runs. The remaining terms in (12), (13) and (14) are of order $10^{-4} N_{0}$ or smaller and are treated as small correction terms for which calculated values of the $\left(V_{0, j} / V_{0,1}\right)$ are again used and for which it is sufficient to use only approximate values for the derivatives. Taking the logarithm of (11) to get

$$
\begin{gathered}
\ln P_{i}-\ln P_{i+1}=\ln Z_{i}-\ln Z_{i+1}+\ln N_{0}+ \\
{\left[\text { corrections }<10^{-4}\right]}
\end{gathered}
$$

yields the working equation which was used, together with eq (2) in the analysis of the lower pressure Burnett data. Densities at the higher pressures in the Burnett measurements are obtained from the lower pressure densities of that run using the analogue of eq (3), which for this apparatus becomes

$$
\rho_{i}=\rho_{i+1} N_{i}^{\prime},
$$

where $N_{i}^{\prime}$ is an effective volume ratio given by

$$
N_{i}^{\prime}=\frac{N_{i}+\left.\frac{1}{\rho_{i+1}} \frac{\partial \rho_{i+1}}{\partial T}\right|_{p} \sum_{i=1}^{n} \frac{V_{i+1, j}}{V_{i, 1}} \Delta T_{i+1, j}}{1+\left.\frac{1}{\rho_{i}} \frac{\partial \rho_{i}}{\partial T}\right|_{P} \Delta T_{i, 1}} .
$$

\section{Data for Propane}

Numerous PVT data for propane up to $325^{\circ} \mathrm{C}$ have been published. To verify the performance of the apparatus and to confirm the accuracy of the data analysis, limited measurements on propane to $325^{\circ} \mathrm{C}$ have been made and have been compared with the literature values. Propane samples were research grade certified to be of $99.99 \%$ purity.

The two largest cell volumes were used for the expansions. Four expansion runs at $250^{\circ} \mathrm{C}$ using helium were first made from a maximum pressure of about $5 \mathrm{MPa}$ to a minimum pressure of about $0.13 \mathrm{MPa}$ in order to derive the cell constant $\mathrm{N}_{0}$. A value of $\mathrm{N}_{0}=1.60702 \pm .0001$ resulted from the analysis of pressure ratios using eq (8) and this value was used in the propane analysis.

Three expansions runs on propane at $250^{\circ} \mathrm{C}$, consis- 
ting of a total of 30 pressure observations, were made [7]. Pressures ranged from a maximum of about $35 \mathrm{MPa}$, corresponding to a density of about 1.6 times the critical density, to a minimum of about $0.14 \mathrm{MPa}$.

The data are separated into two groups for analysis. At lower pressures, corresponding to densities less than the critical density, the data from all runs could be analyzed simultaneously using an expression for the compressibility $Z$ in terms of pressure [8]. Since it is the nature of Burnett experiments to concentrate the data at the lower pressures, only the highest pressure point on each run could not be handled in this manner.

For data below $17 \mathrm{MPa}$ the compressibility factor $Z$ was represented as

$$
Z=1+\sum_{i=1}^{n} B_{i}\left(\frac{P}{P_{0}}\right)^{i}
$$

where $P_{0}=17 \mathrm{MPa}$ and was used with eq (15) for fitting purposes. Data were fitted, in the sense of least squares, by minimization of the difference between the left and right sides of eq (15). For pressures below 1.3 MPa, differences were weighted by $P^{-1}$ to reflect the decreasing relative accuracy of the pressure balance at the lower pressures [8]. Other data were assigned a weight of 1 . However, the results of the fit were insensitive to the weighting. For simplicity, derivatives appearing in the small correction terms of eq (15) were evaluated from a recently formulated correlation of propane data [9]. In the absence of derivative data, however, an interative procedure can be used with equally good results. In this case a best estimate of the derivatives, based on a corresponding states approach, for example, could be used, or the correction terms could be ignored in first approximation. At the completion of the experiments, when the isochoric data are available, the derivatives can be estimated and successive iterations performed. Coefficients for eq (18) are shown in table 1. Burnett density results are tabulated in table 2 along with the densities obtained gravimetrically during the isochoric measurements.

It must be emphasized that eq (18) is simply a fitting function, and its coefficients cannot be identified with the virial coefficients, although the coefficient of the

Table 1. Coefficients of equation (18).

\begin{tabular}{l}
\hline $\mathrm{B}_{1}=-4.14084758401 \mathrm{E}-01$ \\
$\mathrm{~B}_{2}=0$ \\
$\mathrm{~B}_{3}=0$ \\
$\mathrm{~B}_{4}=4.01931812102 \mathrm{E}-01$ \\
$\mathrm{~B}_{5}=-2.20024107867 \mathrm{E}-01$ \\
$\mathrm{~B}_{6}=9.14412474575 \mathrm{E}-03$ \\
$\mathrm{~B}_{7}=4.22392090629 \mathrm{E}-02$ \\
\hline
\end{tabular}

Table 2. Densities along the $250^{\circ} \mathrm{C}$ isotherm obtained from the Burnett measurements and gravimetrically (the latter marked with*).

\begin{tabular}{lccc}
\hline \hline$P(\mathrm{MPa})$ & $\rho\left(\mathrm{mol} / \mathrm{dm}^{3}\right)$ & $P(\mathrm{MPa})$ & $\rho\left(\mathrm{mol} / \mathrm{dm}^{3}\right)$ \\
\hline 34.614 & 7.857 & 3.712 & 0.937 \\
33.953 & $7.784^{*}$ & 3.501 & 0.879 \\
28.960 & $7.241^{*}$ & 2.962 & 0.734 \\
25.118 & $6.714^{*}$ & 2.390 & 0.583 \\
22.311 & 6.252 & 2.250 & 0.547 \\
20.322 & 5.864 & 1.894 & 0.456 \\
20.277 & $5.843^{*}$ & 1.521 & 0.363 \\
16.261 & 4.891 & 1.430 & 0.341 \\
12.922 & 3.891 & 1.200 & 0.284 \\
12.719 & $3.827^{*}$ & 0.960 & 0.226 \\
12.185 & 3.649 & 0.902 & 0.212 \\
10.384 & 3.044 & 0.755 & 0.177 \\
8.536 & 2.421 & 0.603 & 0.141 \\
8.083 & 2.271 & 0.566 & 0.132 \\
6.922 & 1.895 & 0.473 & 0.110 \\
5.674 & 1.507 & 0.354 & 0.082 \\
5.364 & 1.413 & 0.221 & 0.051 \\
4.566 & 1.179 & & \\
\hline
\end{tabular}

linear term is in reasonable agreement with published second virials for propane at $250^{\circ} \mathrm{C}[10,11]$.

Densities for the highest pressures on each of the three runs were calculated from the next lower density of that run using eq (16).

Pressure measurements were made as a function of temperature at fixed total amounts of fluid using the isochoric system to extend the temperature range. Pressures were measured along 10 pseudo-isochores covering a density range of 1.7 to $7.7 \mathrm{~mol} / \mathrm{dm}^{3}$. Observations were made at $10^{\circ} \mathrm{C}$ intervals up to $150^{\circ} \mathrm{C}$ and at $25^{\circ} \mathrm{C}$ intervals up to $325^{\circ} \mathrm{C}$ and to a maximum pressure of about $34 \mathrm{MPa}$. For isochores intersecting the $250^{\circ} \mathrm{C}$ isotherm below $8.5 \mathrm{MPa}$ the densities assigned to the pseudo-isochores were calculated from the intersection pressures using eq (18).

Since the data are concentrated at the lower pressures, the density data above $8.5 \mathrm{MPa}$ were too widely spaced to permit accurate interpolation. To fill in these gaps several isochoric runs were terminated by condensing the samples into a cell held at liquid nitrogen temperature for the purpose of weighing. Sample weights were determined to better than \pm 0.01 gram and densities at $250^{\circ} \mathrm{C}$ were calculated using the volume calibration data for the isochoric system. Uncertainty in densities determined gravimetrically is estimated at better than $\pm 0.1 \%$ due primarily to the uncertainty in volume calibrations. These densities are tabulated in table 2 along with the Burnett densities. Densities for pressures above $8.5 \mathrm{MPa}$, determined by both the Burnett and gravimetric methods, were used in a polynominal fit of pressure versus density, which in general represented 
the densities to better than $0.05 \%$. This function was used to calculate the densities assigned to the pseudoisochores intersecting the $250^{\circ} \mathrm{C}$ isotherm above $8.5 \mathrm{MPa}$.

In calculating the densities along the pseudoisochores, corrections were made for fluid residing in the piping, valve, and the pressure transducer as well as for the effects of thermal expansion and pressure dilation of the cell. Data along the pseudo-isochores are presented in table 3.

Density data along several isotherms are compared with the literature data [11-14] in figure 3. Density deviations are plotted relative to an equation of state for propane [9] used as a baseline. This was done to eliminate the need for multiple interpolations when comparing data at slightly different temperatures. Absolute deviations in figure 3 reflect only the ability of the equation of state to reproduce the experimental data. Excellent agreement between data sets is indicated, however, by the fact that all the experimental data lie in a band of about $\pm 0.1 \%$ which is well within the combined experimental errors. The excellent agreement between closely spaced densities along the $250^{\circ} \mathrm{C}$ isotherm obtained in the Burnett measurements, the gravimetric measurements and from the literature data verify the performance of the experimental apparatus and confirm an estimated accuracy of about $\pm 0.1 \%$ for the Burnett data.

\section{References}

[1] Burnett, E. S. Compressibility determinations without volume measurements. J. Appl. Mech. 3: A136; 1936.

[2] Pope, G. A.; Patsy S. Chappeleav and Kiki Kobayashi. Virial coefficients of argon, methane and ethane at low reduced temperatures. J. Chem. Phys. 59: 423; 1973.

[3] Only general operating characteristics are discussed. Lengthy descriptions of electronic instruments, logic circuitry and computer software have been omitted. It is this author's opinion that these elements, if not standard, are unique to a given apparatus and that each experimenter tends to develop these systems independently. More specific information can be obtained through personal communication.

[4] Hall, K. R., and F. B. Canfield. Optimal recovery of virial coefficients from experimental compressibility data. Physica 33: $481 ; 1967$

[5] Hall, K. R., and F. B. Canfield. A least-squares method for reduction of Burnett data to compressibility factors and virial coefficients. Physica 47: 99; 1970.

[6] Wielopolski, P., and W. Warowny. A fast least-squares method for reduction of Burnett data to compressibility factors and virial coefficients. Physica 91A: 66; 1978.

[7] Due to the many corrections that are made to the raw experimental data in the analysis, only analyzed data are given here. Raw data can be obtained from the author through personal communication.
Table 3. Propane densities along pseudo-isochores.

\begin{tabular}{|c|c|c|c|c|c|}
\hline$P(\mathrm{MPa})$ & $T\left({ }^{\circ} \mathrm{C}\right)$ & $\rho\left(\mathrm{mol} / \mathrm{dm}^{3}\right)$ & $P(\mathrm{MPa})$ & $T\left({ }^{\circ} \mathrm{C}\right)$ & $\rho\left(\mathrm{mol} / \mathrm{dm}^{3}\right)$ \\
\hline 5.191 & 99.999 & 7.314 & 5.606 & 119.994 & 3.855 \\
\hline 6.707 & 109.988 & 7.308 & 6.175 & 129.995 & 3.853 \\
\hline 8.260 & 119.996 & 7.302 & 6.741 & 140.000 & 3.850 \\
\hline 9.831 & 130.004 & 7.299 & 7.296 & 149.998 & 3.848 \\
\hline 11.416 & 140.000 & 7.294 & 8.678 & 174.998 & 3.843 \\
\hline 13.003 & 149.938 & 7.288 & 10.041 & 199.994 & 3.836 \\
\hline 17.003 & 174.979 & 7.275 & 11.388 & 225.003 & 3.830 \\
\hline 21.015 & 199.993 & 7.262 & 12.719 & 249.987 & 3.824 \\
\hline 24.993 & 224.992 & 7.250 & 14.040 & 275.034 & 3.817 \\
\hline 28.960 & 249.993 & 7.239 & 15.341 & 299.905 & 3.811 \\
\hline 5.570 & 110.003 & 5.899 & 16.635 & 324.943 & 3.805 \\
\hline 6.587 & 119.998 & 5.894 & 7.470 & 129.999 & 5.734 \\
\hline 7.620 & 129.999 & 5.890 & 8.467 & 140.023 & 5.730 \\
\hline 8.662 & 139.992 & 5.888 & 9.468 & 150.001 & 5.728 \\
\hline 9.712 & 150.016 & 5.884 & 11.983 & 175.014 & 5.718 \\
\hline 12.345 & 175.000 & 5.873 & 14.498 & 200.018 & 5.708 \\
\hline 14.981 & 199.999 & 5.863 & 17.009 & 225.033 & 5.698 \\
\hline 17.613 & 224.996 & 5.853 & 19.504 & 249.980 & 5.689 \\
\hline 20.227 & 249.985 & 5.843 & 21.985 & 275.002 & 5.680 \\
\hline 4.180 & 89.991 & 7.875 & 24.449 & 300.021 & 5.671 \\
\hline 5.950 & 100.002 & 7.868 & 26.891 & 325.023 & 5.662 \\
\hline 7.768 & 109.998 & 7.861 & 4.866 & 119.988 & 2.653 \\
\hline 9.614 & 119.994 & 7.858 & 5.217 & 129.989 & 2.651 \\
\hline 11.477 & 130.004 & 7.852 & 5.564 & 140.030 & 2.649 \\
\hline 13.350 & 140.007 & 7.845 & 5.905 & 149.996 & 2.648 \\
\hline 15.230 & 149.991 & 7.839 & 6.744 & 175.023 & 2.644 \\
\hline 19.941 & 175.006 & 7.825 & 7.566 & 200.039 & 2.640 \\
\hline 24.646 & 200.015 & 7.812 & 8.374 & 225.034 & 2.636 \\
\hline 29.319 & 225.001 & 7.799 & 9.169 & 249.968 & 2.632 \\
\hline 33.953 & 250.005 & 7.787 & 9.955 & 274.960 & 2.628 \\
\hline 4.804 & 100.000 & 6.783 & 10.734 & 299.980 & 2.624 \\
\hline 6.082 & 110.002 & 6.778 & 11.504 & 325.041 & 2.619 \\
\hline 7.397 & 119.996 & 6.772 & 5.134 & 119.995 & 3.013 \\
\hline 8.735 & 129.999 & 6.770 & 5.549 & 130.011 & 3.011 \\
\hline 10.087 & 139.996 & 6.765 & 5.957 & 140.011 & 3.009 \\
\hline 11.449 & 150.021 & 6.760 & 6.359 & 149.976 & 3.007 \\
\hline 14.869 & 174.997 & 6.747 & 7.354 & 174.989 & 3.003 \\
\hline 18.298 & 199.996 & 6.735 & 8.323 & 199.998 & 2.998 \\
\hline 21.721 & 225.006 & 6.724 & 9.282 & 225.023 & 2.994 \\
\hline 25.118 & 250.006 & 6.713 & 11.160 & 274.984 & 2.984 \\
\hline 28.498 & 275.021 & 6.703 & 12.084 & 300.032 & 2.980 \\
\hline 31.853 & 300.032 & 6.693 & 12.996 & 324.977 & 2.975 \\
\hline 6.020 & 120.012 & 4.805 & 3.722 & 110.000 & 1.779 \\
\hline 6.779 & 130.006 & 4.802 & 3.935 & 120.001 & 1.778 \\
\hline 7.539 & 140.011 & 4.798 & 4.145 & 130.004 & 1.777 \\
\hline 8.298 & 150.001 & 4.795 & 4.352 & 140.009 & 1.776 \\
\hline 10.192 & 175.012 & 4.789 & 4.556 & 150.001 & 1.775 \\
\hline 12.073 & 199.970 & 4.781 & 5.058 & 174.999 & 1.772 \\
\hline 13.946 & 224.994 & 4.772 & 5.550 & 199.996 & 1.770 \\
\hline 15.801 & 250.005 & 4.764 & 6.035 & 224.995 & 1.767 \\
\hline 17.642 & 274.943 & 4.756 & 6.512 & 249.994 & 1.765 \\
\hline 19.462 & 299.904 & 4.749 & 6.984 & 275.029 & 1.762 \\
\hline 21.282 & 324.917 & 4.741 & 7.449 & 299.971 & 1.760 \\
\hline 4.443 & 99.998 & 3.860 & 7.911 & 325.020 & 1.757 \\
\hline 5.031 & 109.999 & 3.858 & & & \\
\hline
\end{tabular}


Figure 3-Comparison of density data from various sources: this work, Beattie (ref. 12), Thomas (ref. 11), Warony (ref. 13), Tiechman (ref. 14).

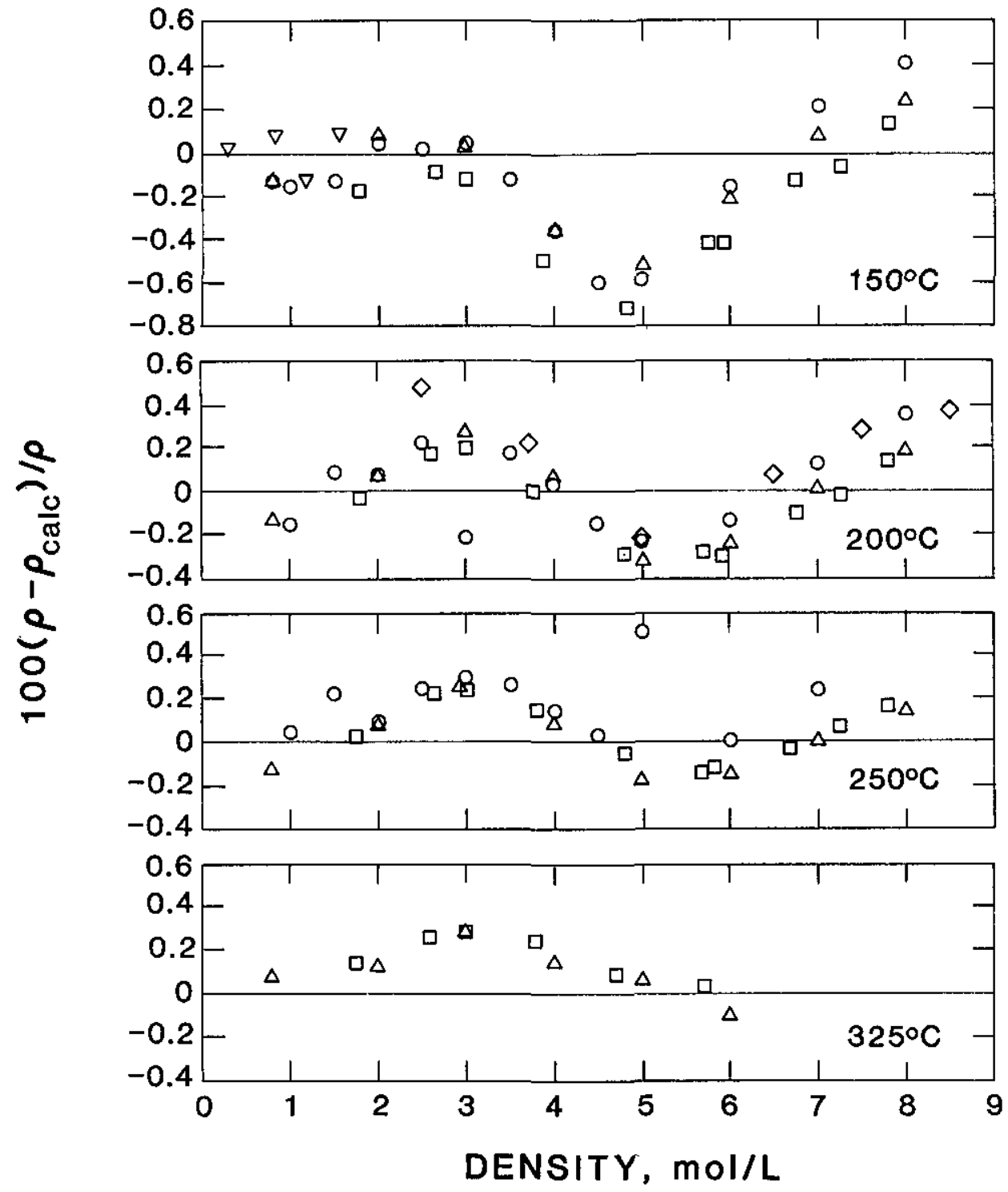

[8] Kell, G. S. Experimental error of the Burnett experiment. Physica 105A: 56; 1981.

[9] Ely, J. F., and B. A. Younglove. Personal communication.

[10] See, for example J. H. Dymond and E. B. Smith. The Virial Coefficients of Pure Gases and Mixtures, A Critical Compilation. Oxford: Clarendon Press; 1980.

[11] Thomas, Raymond H. P., and Roland H. Harrison. Pressurevolume-temperature relations of propane. J. Chem. Eng. Data 27: 1; January 1982.

[12] Beattie, J. A.; W. C. Kay and J. Kaminsky. The compressibility of, and an equation of state for, gaseous propane. J. Am. Chem. Soc. 59: 1589; 1937.

[13] Warowny, W.; P. Wielopolski and J. Stecki. Compressibility factors and virial coefficients for propane, propene and their mixtures by the Burnett method. Physica 91A: 73; 1978.

[14] Tiechman, J. Pressure-density-temperature measurements of liquid propane and benzene. Ph.D. dissertation: Ruhr University, Bochum; 1978. 